Data Acquisition and Processing

System at the Movette Laser-Fusion Facility

\author{
J. M. Auerbach \\ J. R. Severyn \\ D. J. Kroepfi
}

This paper was prepared for submittal

to the 1982 Nuclear Science Symposium

Washington, D. C.

October 20-22, 198?

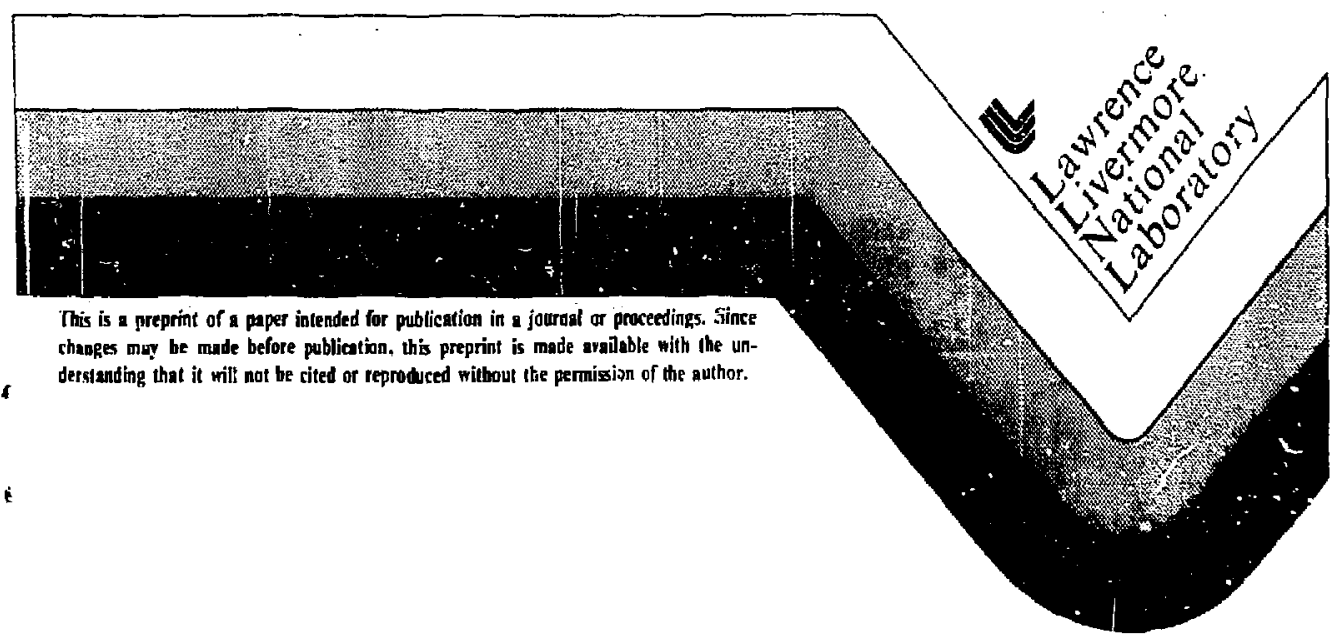


DATA ACQUISITION ANU PROCESSING SYSTEM

J. M. Auerbach, J. R. Severyn, D. J. Kroepf 1

\author{
University of California \\ Lawrence Livermore National Laboratory \\ Livermore, CA 94550
}

Summary

This paper describes the computer hardware and sof tware used for acquisition and processing of data from experiments at the NOVETTE laser fusion facility. Nearly two hundred sensors are used to measure the performance of millimeter extent targets irradiated by multi-kilojoule laser pulses. Sensor output is recorded on CAMAC based digitzers, CCD arrays, and film. CAMAC instrument outputs are acquired and collected by a network of LSI-11 microprocessors centrally controlled by a VAX 11/780. The user controls the systeri through menus presented on color video displays equipped with touch panels. The control VAX cuilects data from a 11 microprocessors and CCD arrays and stores them in a file for transport to a seccnd VAX $11 / 780$ which is used for processing and final analysis. Transfer is done through a high speed fiber-optic link. Relational data bases are used extensively in the processing and archiving of data.

Work performed under the auspices of the U.S. Department of Energy by the Lawrence Livermore National Laboratory under contruct W-7405-Eng-48. 


\section{The Novette Laser Fusion Facility}

The NOVFTTE laser system (Figure i) is a two beam neodymium gluss Iaser with multi-color output capability. It can focus on targets of millimeter extent thousands of joules of either infrared, green, or eventually blue laser light in durations from 100 picoseconds to several nanoseconds. Multicolor capability is achieved with frequency doub? ing crystals at the output of the amplifier chains which produce light with a wavelength of $1.06 \mathrm{H}$ (infrared). Targets for study of inertial confinement fusion and laser-plasma interactions are positioned at the center of an $80 \mathrm{~cm}$ diameter spherical vacuum chamber. The chamber serves as a mount for the large number of diagnostic instruments which record target performance. The laser system and target instrumentation is computer controlled with a VAX 11/780 central computer and a network of LSI-11 microprocessors. The system architecture provides for four functional subsystems:

-Laser Alignment: Control of mirrors, lenses, spatial filter pinholes.

-Power conditioning: control of capacitor banks and firing circuits for laser amplifier flashlamps.

-Laser Diagnostics: monitoring of laser system performance (energy, spatial uniformity, pulse width).

-Target Diagnostics: set-up, control, and recarding of data from target chamber diagnostics and control of the chamber vacuum system. 
The fourth subsystem and a second VAX 11/780 for processing and analysis of diagnostic data will be described in the remainder of this paper.

\section{Target Diagnostics Instrumentation}

Performance of targets is deterimined from the characteristics of enissions from the target. Emission quantities measured inciude:
a. Light scattered and emitted from the target
b. X-rays emitted from the target
c. Particles emitted from the target (ions, neutrons, electrons)

Sensors measuring these emissions can have either spatial, temporal, or spectral resolution or any combination of the three. Sensor output is recorded by $\mathrm{film}$, charge integrators, transient digitizers and $\mathrm{CCD}$ arrays. Film is digitized on a separate system and does not enter the data flow until the second VAX 11/780. The electronic outputs are the only concern in the data acquisition system. They are digitized in either CAMAC compatible instrumentation or in CCD arrays accessed directly by the control VAX computer. Computer control is used to set and monitor integrator sensitivity, digitizer settings, and power supply voitages. 


\section{Diagnostic Station for CAMAC Instrumentation}

A diagnostic station is defined as an eiectrically isolated group of racks, at least one front end processor (LSI-11), at least one CAMAC crate, and the digitizers, amplifiers, power supplies, etc., to support a set of diagnostic sensors. Each station is electricaliy isolated from a11 others and the VAX by using fiber optics for all input and output signals. The fiber optics are part of a local network called NOVANET and are connected to the Node Star as shown in Figure 2. Front End Processor (FEP) and digitizer operation is initiated by a combination of commands

downloaded from the VAX and a trigger system (Figure 3) synchronized with the laser system shot countdown sequence. The slow trigger comes from the power conditioning control system and informs the FEP of the state of the shot sequence. The "slow" system signals are sent tp an Initiator Transmitter where the option of a local dry run capability may be selected. The fiber optic output from the Initiator Transmitter terminates at the Initiator Receiver in the first CAMAC crate of each front end processor at each charge in the shot sequence status and synchonizes the FEP to the shot countdown.

The fast trigger is initiated by a photodiode activated by a part of the laser system oscillator pulse. The slow trigger system allows the fast trigger to take effect only on actual shot sequences. Each FEP nas a fast Trigger Receiver CAMAC module that interrupts the FEP at shot time and also activates all the digitizer modules. Timing experiments are used to synchronize digitizer operation with the arrival of the laser pulse on target. 
Architecture of the Target Diagnastics System

The target diagnostics system architecture is illustrated in Figure 4. Acquisition and control and processing and analysis are separated in two VAX $11 / 780^{\prime} \mathrm{s}$. Each functional area will be described separately.

\section{A. Data Acquisition System Architecture}

The data acquisition and control system is LSI-11 and C.MMAC based except for CCD readouts. It utilizes geographically distributed LSI-11 processors to control various analog to digital converters, anplifiers, and power supplies near the analog signal source of the various diagnostics. This minimizes analog signal cable lengths and maximizes the signal to noise ratio. L\$I-11 processors control a limited number of CAMAC crates so as to allow expansion of the system. Commands to perforn the various operations on the various electronic modules are downloaded from the VAX to the LSI-11. Based on ther commands, the LSI-11's initiate CAMAC controller operations. The user interfaces with the system through a relational data base and menus displayed on color video monitors with options selected through touch panels mounted on the color CRTs. Operators enter the system configuration into the data base through forms presented on video terminal screens. The data base contains the CAMAC location (microprocessor, crate station and unit) cf a11 electronic modules as well as the mapping between diagnostic sensors and power supply channels. This mapping is crucial in the higher level processing since physics data is organized by diagnostic, whereas the 
data acqusition system collects and organizes data by eiectronic readout module. The contents of the configuration data base are transferred to the memory of the control VAX during start-up of the data acquisition system control software.

Architecture of the user interface for control of the data acquisition system is based on a hierarchy of commands, with all but the lowest ieve 1 being defined in terms of previously defined cormanos. At the lowest level are the oasic CAMAC controller commands. At the next level are the basic operational commands (initialize, set_gain, read gain, read_voltages, read_data, etc.) for the various electronic modules (charge integrators, transient digitizers, etc.). These two command leveis are defined at the FEP level and are not normally subject to change. All other commands are defined on the vaX level by means of an ascii file which is read when the system is started. These is no restriction on definition depth and. command may be easily added, deleted, expanded, or otherwise changed. At the uppermost level, commands usually are for glotal operations such as the initialization, testing, or data collection for a group of modules. This is the level used for an experiment. Command execution is the last level of expansion being performed by the FEP.

The configuration data base and detailed command for each type of electronic module are retained at the VAX level. This means minimal intelligence at the front end processor. This philosophy guarantees easy expandability of the system whereas trying to put details of modules at the LSI-11 level would quickly saturate that processor's memory. In that case different processors would have to handle different modules thus eliminating easy interchangability of modules in the CAMAC crates, an unsatisfactory situation for a facility with rapidly changing experiments. 
The user controls and monitars the system with touch panel driven menus presented on RAMTEK color TV displays. The TV screens can also produce high resolution graphics of the data in up to 32 different colors or shades of gray.

Menus allow control and monitoring from the system or electronic module level. Distinct sets of menus and displays are associated with each type of module. As new modules are added, new menus and comnand definitions are correspondingly added to the control system software. Each display allows return to the higher level menus at any time.

B. Architecture of the Higher Level Data Processing System

The target data acquisition system collects data from all CAMAC crates after a shot and stores them in a single file on the control VAX. In addition, each CCD digitized image is stored in an individual file on the VAX. After a shot (experiment), the configuration data base, CAMAC data file, and CCD data files are transmitted to the second VAX 11/780 (Figure 2) through a high speed fiber-optic link utilizing NOVANET hardware and software. Data transfer through the link is one-way, as security requirements exist for the final analyzed data. Storage media for the data files are high capacity disk drives with the archiving medium being magnetic tape.

Processing of the data involves conversion of digitizer and CCO output to relevant physics quantities to insertion in a physics aata base which serves as the data source for scientific analysis, correlations, etc. To implement the processing in a highly automated and efficient manner, the processing architecture relies heavily on the ORACLE relational data base management system. Figure 5 shows the different 
levels of processing. First, using the configuration data base, CAMAC data is organized by diagnostic channel from the CAMAC (FEP, crate, station) organization. Then processing software using settings found in the CAMAC data file converts digitizer output to analog signals (volts vs. time, picocoulombs, etc.). These analog signals are then converted to pnysics parameters using calibration and resuonse characteristics data bases maintained by personnel in charge of the various instruments. With the relational data base, a diagnostic channel can be neatly defined in terins of its components with exchanges and replacements being easily recorded. Implementation of the ORACLE Database query language (SQL) in processing codes allows easy extraction of relevant component parameters and calcilation of calibration parameters in a highly automated manner. This is in contrast to a primitive data base in which many parameter files with frequent editing must be utilized. After analog signals and physics parameters are calculated they are recorded on tables and plots for immediate distribution and stored automatically in a relational data base for archiving and analysis. Experimenters can do data searches and correlations with this data base using the SQL query language.

Image data (both CCO and digitized film data files) are anajyzed with a wide variety of image processing software. A RAMTEK color graphics system produce color enhanced images and plots of lineouts of the data. Sof tware also provides for deconvolution of film and instrument response from the data. 
Conclusion

Over a inegabyte of data and nearly two hundred diagnostics can be associated with a single experiment on NOVETTE. Diagnostics can change from experiment to experiment requiring detailed configuration control and recording.

Timely set-up of an experiment and timely processing of data require an expandable, maintainable and highly automated data acquisition and processing computer system. The use of distributed front end processors, high speed fiber optic links, CAMAC standard hardware and a relational data base management system alang with the virtual menory feature of the VAX computers has satisfied this requirement.

\section{DISCLAIVER}

This document was pregared as an account of work sponsored by an agency of the L'nited States Grovemment. Neither the United States Gavernment nor the liniversily of Cadifomia nor uny of their employees, makes any warrany, express or implied, of assomes any lepal lisbility or responsibility for the accuracy, completeness, of uselulness of any information, apparatus, product, or process disclosed. of represents that its use would not infringe privaley owned rights. Reference hereitt to any specific cammercisl products, prosess, or service hy trade name, trademark, manufacturer, or otherwise, does not necessarily constitute or imply ils endorsement, necommendation, or furoring by the Inited Sitates Ciavernmert ar the Universily of Califomia. The views and opinions of auhars exprest herein do wot necessarily state or rellet those al the Linited States Coverute as thered, sod shall aot be ased for adyetising or product ent domement porpors. 


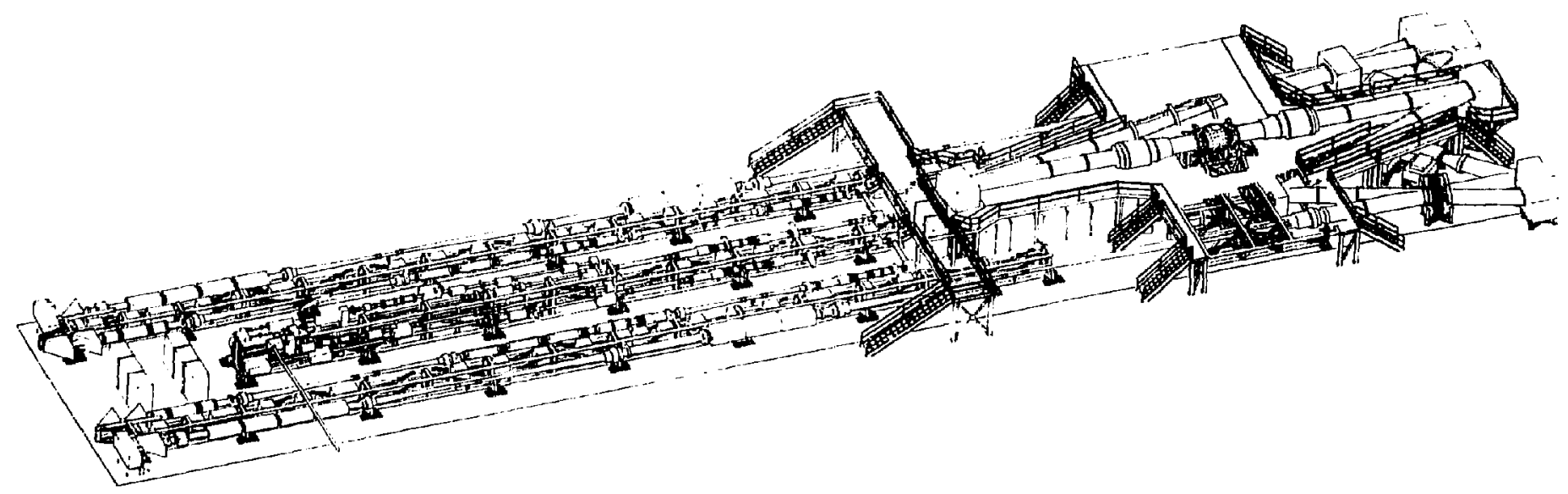

02-32-0282-0655C 


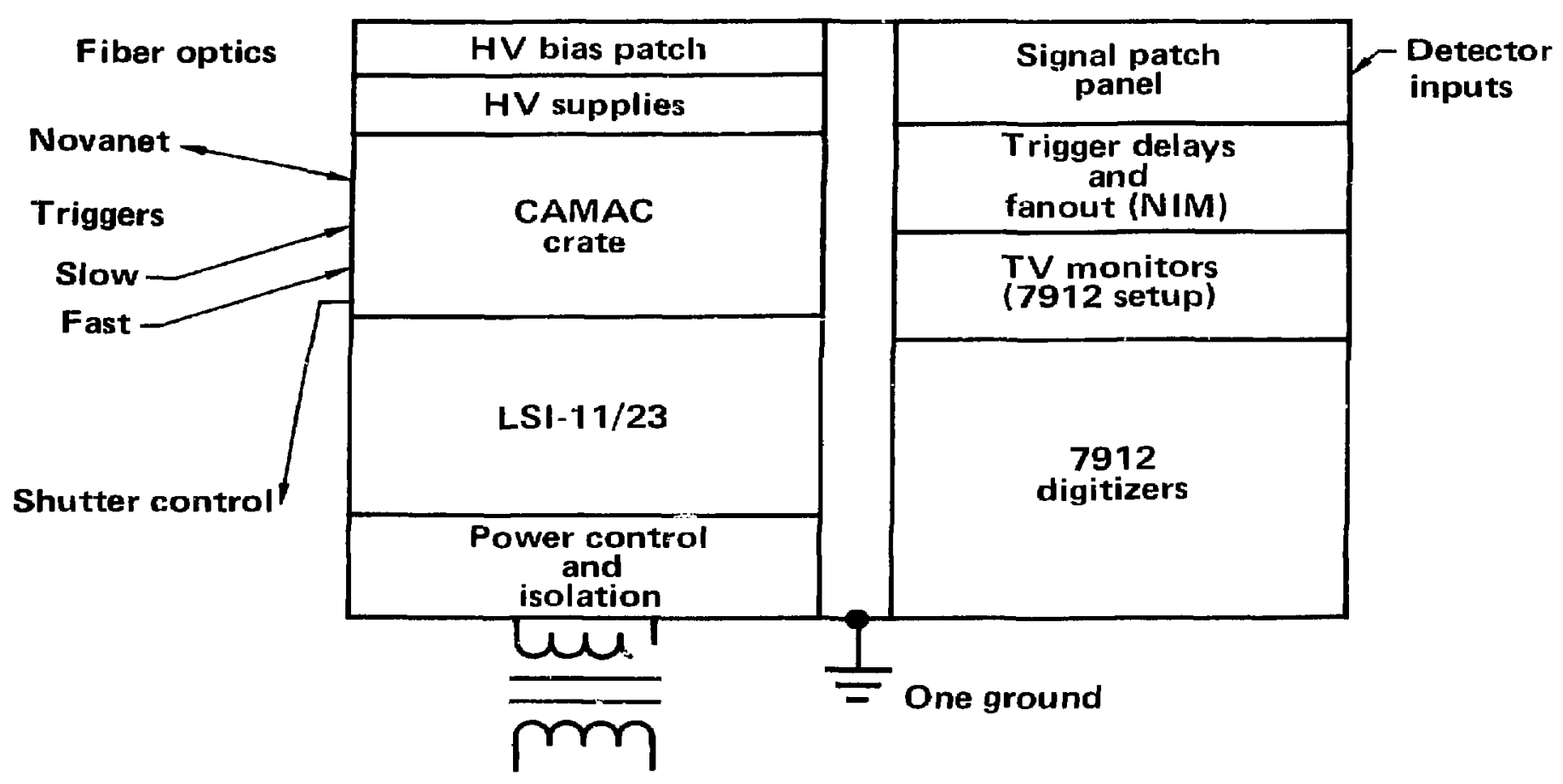




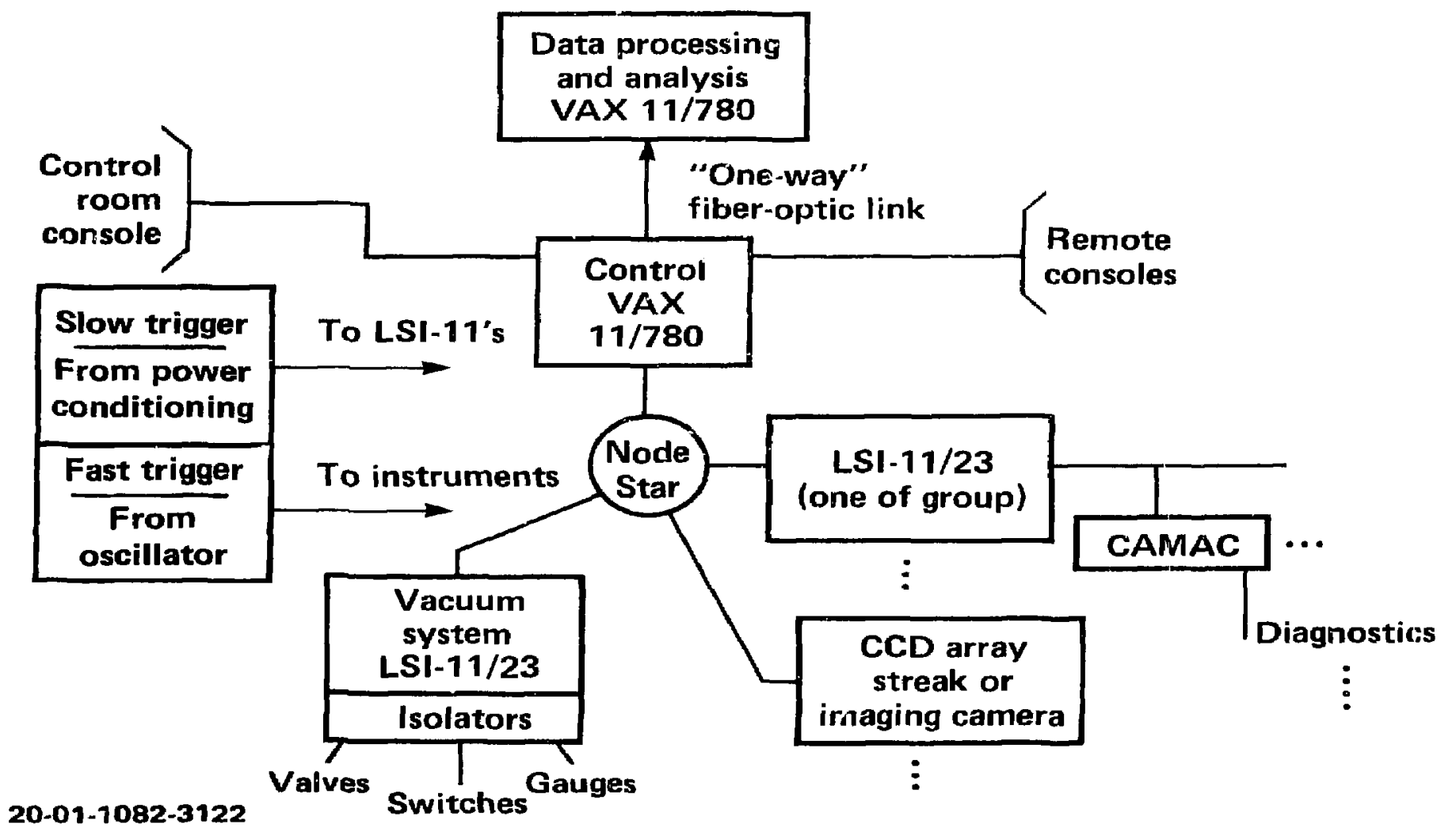


PROCESSING OF EXPERIMENTAL DATA

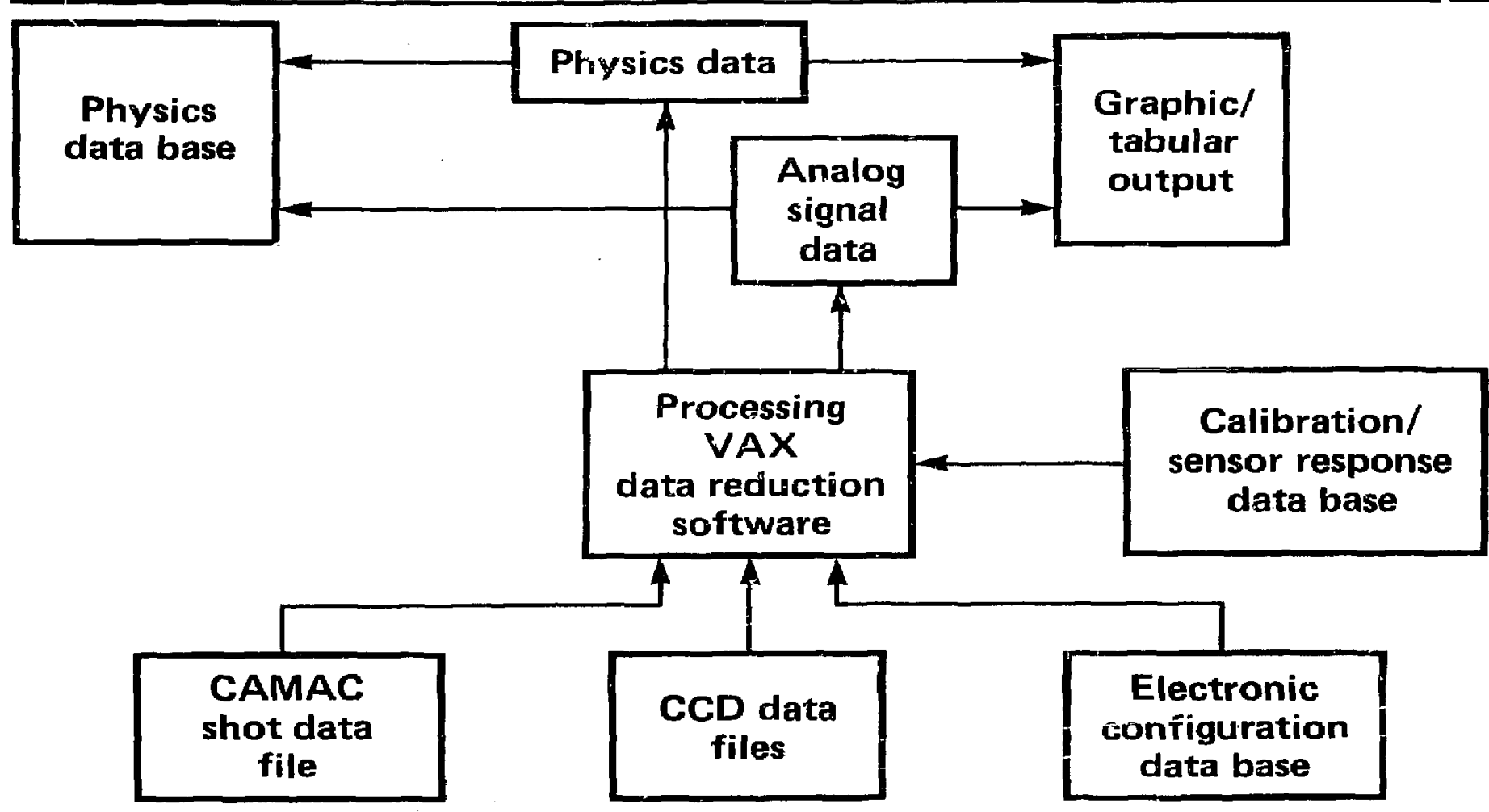

